though we are certainly not advocating conservative management of transmediastinal GSWs, survival might be possible (rarely).

\section{References}

1. Johnson SB, Nielsen JL, Sako EY, Calhoon JH, Trinkle JK, Miller OL. Penetrating intrapericardial wounds: clinical experience with a surgical protocol. Ann Thorac Surg. 1995;60:117-21.
2. Wall MJ Jr, Mattox KL, Chen CD, Baldwin JC. Acute management of complex cardiac injuries. J Trauma. 1997;42:905-12.

3. Moreno C, Moore EE, Majure JA, Hopeman AR. Pericardial tamponade: a critical determinant for survival following penetrating cardiac wounds. J Trauma. 1986;26:821-5.

4. Kulshrestha P, Das B, Iyer KS, Sampath KA, Sharma ML, Rao IM, et al. Cardiac injuries—a clinical and autopsy profile. J Trauma. 1990;30: 203-7.

\title{
Bilateral lung transplantation for pulmonary hypoplasia caused by congenital diaphragmatic hernia
}

\author{
Richard Lee, MD, Eric N. Mendeloff, MD, Charles Huddleston, MD, Stuart C. Sweet, MD, PhD, and \\ Maite de la Morena, MD, St Louis, Mo
}

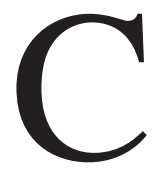

ongenital diaphragmatic hernia $(\mathrm{CDH})$ remains a lifethreatening neonatal anomaly. Patients who have $\mathrm{CDH}$ without pulmonary hypoplasia have an expected mortality that ranges from $10 \%$ to $25 \% .^{1}$ However, when pulmonary hypoplasia is associated with $\mathrm{CDH}$, the prognosis is even worse. Although the introduction and use of extracorporeal membrane oxygenation (ECMO) has improved survival in patients with unilateral pulmonary hypoplasia, mortality still ranges from $20 \%$ to $40 \%$ in this group. ${ }^{1} \mathrm{CDH}$ with bilateral pulmonary hypoplasia is a condition that has previously been incompatible with life.

Several innovative therapies have been offered in an attempt to improve the outcome in these high-risk patients. One of these therapies is lung transplantation. This option is often discussed as a potential solution and has some experimental support. ${ }^{2,3}$ However, the clinical experience in the literature is limited to a case report of a unilateral lung transplantation, ${ }^{4}$ followed by a transplant pneumonectomy nearly 5 years after transplantation. ${ }^{5}$ Here we present the outcomes of 3 patients in whom bilateral lung transplantation was performed for pulmonary hypoplasia associated with $\mathrm{CDH}$ at our institution.

\section{Clinical Summaries}

PATIENT 1. A diagnosis of $\mathrm{CDH}$ was established at 18 weeks' gestation in a female fetus. An ultrasound demonstrated the stom-

\footnotetext{
From the Division of Cardiothoracic Surgery and the Department of Pediatrics, Washington University, St Louis, Mo.

Received for publication Sept 7, 2002; accepted for publication Dec 2, 2002.

Address for reprints: Richard Lee, MD, Department of Thoracic and Cardiovascular Surgery, Cleveland Clinic Foundation, 9500 Euclid Ave, Desk F-24, Cleveland, OH 44195 (E-mail: leer2@ccf.org).

J Thorac Cardiovasc Surg 2003;126:295-7

Copyright (C) 2003 by The American Association for Thoracic Surgery $0022-5223 / 2003 \$ 30.00+0$

doi:10.1016/S0022-5223(03)00202-2
}

ach, bowel, and part of the left lobe of the liver in the left chest and the heart in the right chest. Displacement of the heart made evaluation difficult. Shortly after birth, the child was blue and without respiratory effort. Initial physical examination was notable for absent breath sounds on the left, as well as heart sounds on the right. She was immediately intubated. Chest radiography demonstrated viscera in the left chest. Echocardiography demonstrated double-inlet left ventricle, transposition of the great arteries, subaortic stenosis, coarctation of the aorta, a patent ductus arteriosus, and a patent foramen ovale with left-to-right flow.

The patient's condition was extremely difficult to stabilize. Four hours after birth, ECMO was started. Epoprostenol (prostaglandin $\mathrm{E}_{1}$ ) was infused to maintain patency of the ductus arteriosus. A discussion was held with the family, who agreed that combined heart-lung transplantation would offer the best chance for survival.

On day 19, she underwent combined heart-lung transplantation and repair of the $\mathrm{CDH}$. The left lung was extremely small and the right lung was erythematous and small. Circulatory arrest was used to perform the aortic arch reconstruction. The remainder of the heart-lung transplantation was achieved in standard fashion, and the patient was weaned from cardiopulmonary bypass. Because it appeared that the intrathoracic space was too small for the donor organs, only the skin was closed. The sternum and ribs were left unapproximated. The diaphragmatic hernia was repaired with a large patch. Because the abdomen could not accept all of the viscera, a silo was placed.

Four hours after arrival in the intensive care unit, an episode of electromechanical disassociation prompted 4 minutes of cardiopulmonary resuscitation. Re-exploration only revealed decreased contractility of the right ventricle. ECMO was restarted. The abdominal silo exploration was also unremarkable. On postoperative day 6, day 25 after birth, ECMO was weaned, and the patient was decannulated. The abdomen was closed on day 28. On day 38 , a peritoneal catheter was placed, and peritoneal dialysis was initiated to treat fluid overload that was unresponsive to diuretic therapy. However, this was only marginally successful. Therefore, she was placed on continuous venovenous hemofiltration on day 74. Heparin was infused to maintain activated clotting times of 180 
to 220 seconds. She appeared to initially improve, and the ventilator was slowly weaned. On day 84 after transplantation, the patient had an episode of hypotension and became unresponsive, with fixed and dilated pupils and a full fontanel. Computed tomographic scanning showed a large subarachnoid blood collection with a midline shift. Support was withdrawn, and the patient died.

PATIENT 2. A diagnosis of congenital left diaphragmatic hernia was established by ultrasonography at 27 weeks' gestation. The child was delivered by cesarean section after late fetal heart decelerations into the 60 -beats/min range, with a birth weight of $3.2 \mathrm{~kg}$. Because of severe respiratory distress and cyanosis, he was intubated. Initial physical examination was notable for absent breath sounds on the left and a point of maximal impulse displaced to the right. A chest radiograph demonstrated bowel throughout the left hemithorax.

Although the patient initially had adequate oxygenation, his condition rapidly deteriorated over the first 12 hours after birth. Despite maximal ventilator settings, he continued to have poor oxygenation, with evidence of pulmonary hypertension. Twelve hours after birth, ECMO was started. A tube was placed in the right side of the chest for a pneumothorax. On day 12 after birth, the chest tube began to have high-volume, bloody outputs that prompted a surgical exploration, followed by repair of the diaphragmatic hernia. On day 15 , because of positive cultures from the ECMO circuit, he was decannulated and placed on an oscillating ventilator. His course was complicated by recurrent pneumothorax requiring bilateral tube thoracostomies at 20 days after birth. Attempts at conventional ventilation were unsuccessful.

Because of progressive respiratory and hemodynamic instability, ECMO was restarted on day 25. When it appeared that his lungs would not support him, he was listed for lung transplantation. Bilateral lung transplantation was performed on day 36 after birth. The native right lung had multiple air leaks and 2 obvious areas of necrosis. Initially, an attempt was made to transplant only the left lung. However, a decline in oxygenation after cardiopulmonary bypass was terminated prompted transplantation of the right lung as well. The patient was then successfully weaned from cardiopulmonary bypass. He did not tolerate chest closure; therefore, the chest was left open, and a silicone rubber mesh cover was sewn over it. A postoperative perfusion scan demonstrated no flow to the left pulmonary artery, necessitating a return to the operating room for an anastomotic revision. This was due to a kink in the left pulmonary artery anastomosis associated with complete inflation of the left lung. Because of persistent hemodynamic instability, ECMO was started for the repair. However, he could not be weaned from ECMO. After discussion with the family, he was decannulated from ECMO on day of life 51, and despite high-dose inotropic support, he did not survive.

PATIENT 3. In this patient a diagnosis of $\mathrm{CDH}$ was established at 18 weeks' gestation. Shortly after birth, this $2.8-\mathrm{kg}$ girl gave out a weak cry, and severe cyanosis developed. Because of severe respiratory distress, she was intubated and placed on mechanical ventilation. Initial physical examination was notable for absent breath sounds on the left, as well as an imperforate anus. A chest radiograph demonstrated bowel throughout the left hemithorax.

The patient was extremely difficult to oxygenate. Despite maximal oscillator ventilator settings, she continued to have poor oxygen saturation and evidence of pulmonary hypertension. In addition, she required inotropic support to maintain her blood pressure. ECMO was started 12 hours after birth. The imperforate anus was managed by dilatation of the posterior forchette fistula. On day 4 after birth, the $\mathrm{CDH}$ was repaired with a polytetrafluoroethylene patch during ECMO. This was complicated by a reexploration for bleeding on day 5. A slow ECMO wean was preformed, and she was decannulated from ECMO on day 14. After successful treatment of an episode of sepsis, a colostomy and mucous fistula were placed on day 40. She was unable to be weaned from the ventilator, and a tracheostomy was performed. Echocardiography suggested worsening pulmonary hypertension, and the patient began to exhibit worsening oxygenation. On day 83, a cardiac catheterization revealed suprasystemic pulmonary artery pressures with minimal response to oxygen and nitric oxide. She was listed for lung transplantation and maintained on dopamine, prostacyclin, nitric oxide, sedation, and paralysis. She underwent bilateral sequential lung transplantation on her 105th day in the hospital. During transplantation, the donor left pulmonary artery was anastomosed to the main pulmonary artery because of a diminutive left pulmonary artery in the recipient.

After lung transplantation, she had prolonged ventilation. By day 174 , she achieved respiratory independence. On day 185 , the tracheostomy tube was removed. On day 187, 82 days after her lung transplantation, she was discharged home. Her course was complicated after discharge by 2 readmissions for treatment of $\alpha$-hemolytic streptococcal pneumonia 6 and 10 months after transplantation. At 10 months of age, she underwent a Nissen fundoplication to treat severe reflux. After 1 year, she underwent a posterior sagittal anorectoplasty. Currently, the patient remains alive and well and is walking at the age of 2 years 9 months. Infant pulmonary function tests have shown a gradual increase in the functional residual capacity, a surrogate for vital capacity. This has risen from $110 \mathrm{~mL}$ to $155 \mathrm{~mL}$ over the 2-year period of follow-up: the volume per kilogram has remained stable at 15 to $16 \mathrm{~mL} / \mathrm{kg}$, with the normal range being 15 to $25 \mathrm{~mL} / \mathrm{kg}$. Although this is not conclusive evidence of lung growth parallel to somatic growth, it is the best evidence we can obtain in small infants undergoing lung transplantation. During this period of follow-up, she has undergone 8 transbronchial biopsies as surveillance for rejection and thus far has had no evidence of rejection.

\section{Discussion}

The experience with pediatric lung transplantation is limited. Overall, 587 pediatric patients have undergone lung transplantation, with a 1-year survival of $75 \%$. Lung transplantation in infants is uncommon. In our institution we have performed 210 lung transplantations in children under the age of 18 years; 32 patients were under the age of 1 year at the time of transplantation.

The only previously published report of lung transplantation for $\mathrm{CDH}$ was a clinical success. ${ }^{4}$ In that case the recipient received a single right lung on day 17 after birth, 8 days after ECMO decannulation. The small size of the recipient chest necessitated a right middle lobectomy from the donor. On postoperative day 57, she was weaned from the ventilator. On postoperative day 90 , she was discharged home. At age 4 years 10 months, a transplant pneumonectomy was performed because of side effects of immunosuppression and poor function of the transplanted lung. 
Here we detail our experience of 3 patients who received bilateral lung transplants for pulmonary hypoplasia in association with $\mathrm{CDH}$, one of whom received a combined heart-lung transplant because of coexisting severe congenital heart disease. Although no conclusions can be drawn from this small experience, several interesting observations arise.

Of the 4 reported patients, 2 died. It is notable that the 2 patients who were weaned from ECMO before transplantation survived. This might reflect that the patients had organ failure limited to a single system at the time of transplantation. In contrast, both patients who underwent transplantation during ECMO died. Our overall experience with ECMO as a bridge to transplantation includes 18 patients: 4 died before transplantation; 6 were weaned from ECMO and subsequently undertent transplantation, with 4 long-term survivors; and 8 underwent transplantation during ECMO, with 1 long-term survivor. Although there is an obvious survival advantage to those weaned from ECMO before transplantation, it might not be possible to predict the likelihood of ECMO weaning when this is initiated. All these operations are technically difficult because of the small hilar structures of the hypoplastic lung. The pulmonary artery anastomosis is probably best placed into the main pulmonary artery in light of the problems noted in our patient 2 above. The bronchus on that side is often very small as well. It is probably best to repair the diaphragmatic hernia early in the course of these patients. The presence of a significant volume of the abdominal contents in the chest prevents expansion of the abdominal cavity sufficient to accommodate these organs. This increases the likelihood of leaving the abdominal wound open with a silo after transplantation, as well as prolonging the time in the operating room for the procedure.

It is unclear whether single- or double-lung transplantation is most appropriate for these patients. The most attractive option is a single-lung transplant on the side of the hernia, allowing the patient to survive early infancy. After satisfactory growth and development of the native lung on the other side, it should be possible to remove the transplanted lung and stop all immunosuppression, as was the case reported by DeAnda and colleagues. ${ }^{5}$ It is very difficult to judge the adequacy of the contralateral lung before transplantation because it has been subjected to some degree of barotrauma and high levels of inhaled oxygen before transplantation. It is very unlikely to be normal: the patient would likely not require transplantation under those circumstances. One could perform single-lung transplantation on the affected side, try to wean from cardiopulmonary bypass, and transplant the other lung if that fails. We accept donors with body lengths $25 \%$ above and below that of our recipient. Admittedly, this is an arbitrary figure, but it has worked out on a practical level. Extending this range upward is feasible with a plan to use the lower lobes only; this extends the donor length range to 2 or 3 times that of the recipient. One must bear in mind that the chest wall edema that occurs as a consequence of prolonged ECMO time will compromise organ implantation, even with a perfect size match.

Lung transplantation for pulmonary hypoplasia associated with $\mathrm{CDH}$ is a treatment modality without extensive clinical experience. It does represent a viable option for survival in patients who would otherwise be ventilator dependent at best. It is difficult to accurately predict which patients will require lung transplantation early in life. Once a patient is started on ECMO, there is very little time to safely wait for a suitable donor. Given the observed morbidity and mortality to date in this limited experience, it might be advisable to reserve transplantation for those patients able to be weaned from ECMO but remaining critical with persistent pulmonary hypertension on maximal ventilatory support. Early consultation with a lung transplant center and identification of appropriate candidates will remain a key factor in successful application of lung transplantation for these very ill infants.

\section{References}

1. Butler MW, Stolar CJH, Altman RP. Contemporary management of congenital diaphragmatic hernia. World J Surg. 1993;17:350-5.

2. Crombleholme TM, Adzick NS, Hardy K, et al. Pulmonary lobar transplantation in neonatal swine: a model for treatment of congenital diaphragmatic hernia. J Pediatr Surg. 1990;25:11-8.

3. Langenburg SE, Blackbourne LH, Buchanan SA, et al. Compensatory growth of porcine right lungs after chronic rejection of transplanted left lungs. Ann Thorac Surg. 1995;59:28-32.

4. Van Meurs KP, Rhine WD, Benitz WE, et al. Lobar lung transplantation as a treatment for congenital diaphragmatic hernia. J Pediatr Surg. 1994:29:1557-60.

5. DeAnda A Jr, Cahill JL, Bernstein D, Starnes VA, Reitz BA. Elective transplant pneumonectomy. J Pediatr Surg. 1998;33:655-6. 